\title{
BMJ Open Evaluation of ADA HbAlc criteria in the diagnosis of pre-diabetes and diabetes in a population of Chinese adolescents and young adults at high risk for diabetes: a cross-sectional study
}

Ge Li, ${ }^{1}$ Lanwen Han, ${ }^{2}$ Yonghui Wang, ${ }^{2}$ Yanglu Zhao, ${ }^{3}$ Yu Li, ${ }^{1}$ Junling Fu, ${ }^{1}$ Ming Li, ${ }^{1}$ Shan Gao, ${ }^{2}$ Steven M Willi ${ }^{4}$

To cite: Li G, Han L, Wang Y, et al. Evaluation of ADA HbA1c criteria in the diagnosis of pre-diabetes and diabetes in a population of Chinese adolescents and young adults at high risk for diabetes: a crosssectional study. BMJ Open 2018;8:e020665. doi:10.1136/ bmjopen-2017-020665

- Prepublication history for this paper is available online. To view these files, please visit the journal online (http://dx.doi. org/10.1136/bmjopen-2017020665).

$M L$ and SG contributed equally. $\mathrm{GL}$ and $\mathrm{LH}$ contributed equally.

Parts of this study was presented in abstract form at the American Diabetes Association's 75th Scientific Sessions, 5-9 June 2015 in Boston, Massachusetts, USA.

Received 16 November 2017 Revised 29 May 2018 Accepted 3 July 2018

Check for updates

(c) Author(s) (or their employer(s)) 2018. Re-use permitted under CC BY-NC. No commercial re-use. See rights and permissions. Published by BMJ.

For numbered affiliations see end of article.

Correspondence to

Professor Shan Gao;

gaoshanmw@163.com

\section{ABSTRACT}

Objective We aimed to assess haemoglobin A1C (HbA1c) for the diagnosis of pre-diabetes and diabetes in a population of Chinese youths at risk of metabolic syndrome.

Setting Beijing, China.

Participants A total of 581 subjects aged 14-28 years underwent evaluation including an oral glucose tolerance test (OGTT). Insulin sensitivity, $\beta$-cell function and a number of cardiovascular disease risk factors were evaluated. Receiver operating characteristic (ROC) curves were used to assess the screening efficacy of HbA1c.

Results Using OGTT data as a standard, the majority $(70.0 \%, 7 / 10)$ of subjects with diabetes would have been diagnosed with $\mathrm{HbA1C} \geq 6.5 \%$. In contrast, only $28.1 \%$ (16/57) of subjects with pre-diabetes possessed elevated HbA1cs, while the majority (68.4\%) had normal HbA1cs. On the contrary, a total of $8.1 \%(39 / 479)$ of youths in the normal $\mathrm{HbA} 1 \mathrm{C}$ category $(<5.7 \%)$ and $21.3 \%$ in the prediabetes category had pre-diabetes. In the ROC analysis, the area under the curve (AUC) for $\mathrm{HbA1C}$ identifying pre-diabetes was $0.680(95 \% \mathrm{Cl} 0.640$ to 0.719$)$; the optimal threshold was $5.5 \%$, with a sensitivity of $61.4 \%$ and specificity of $68.5 \%$. For type 2 diabetes mellitus, the AUC for HbA1c was 0.970 (0.952 to 0.982), and the optimal threshold was $6.1 \%$, with a sensitivity of $90.0 \%$ and a specificity of $98.7 \%$. Applying these new cutoffs, pre-diabetic participants (HbA1c 5.5\%-6.1\%) had lower disposition index and higher risk of dyslipidaemia $(\mathrm{OR}=1.61,95 \% \mathrm{Cl} 1.10$ to 2.37 ) and metabolic syndrome $(\mathrm{OR}=2.09,1.27$ to 3.45$)$ than those with normal HbA1C $(<5.5 \%)$.

Conclusion The American Diabetes Association's established $\mathrm{HbA} 1 \mathrm{C}$ criteria for pre-diabetes and diabetes (5.7\% and 6.5\%) may not be appropriately applied to adolescents and young adults in China. Our findings suggest that those with HbA1c of 5.5\%-6.1\% already exhibit impaired $\beta$-cell function and increased cardiometabolic risk factors which may warrant intervention.

Trial registration number NCT03421444.
Strengths and limitations of this study

- This study included a well-characterised cohort of adolescents and young adults at risk for diabetes.

- All these individuals have undergone an oral glucose tolerance test (OGTT) to evaluate their alterations in insulin sensitivity and $\beta$-cell function.

- This is the first study in a population of Chinese adolescents and young adults to assess the American Diabetes Association (ADA)'s haemoglobin A1C cut-points for predicting diabetes or pre-diabetes against the gold-standard OGTT.

- However, there was a relatively small sample size of individuals with diabetes by ADA criteria in the population of youth.

- While the study cohort was a large population-based sample, it may not be representative of the overall Chinese population as we chose to intensely study a subset at risk for the condition of interest (diabetes/ pre-diabetes).

\section{INTRODUCTION}

The incidence of obesity has increased dramatically in recent decades among Chinese children and adolescents. The Global Burden of Disease Study showed that the prevalence of overweight and obesity in children and adolescents in low-income and middle-income countries has increased from $8.4 \%$ in 1980 to $13.4 \%$ in $2013 .{ }^{1}$ With the global surge in obesity, prevalence of diabetes has increased substantially. WHO data from 2014 estimated that 347 million people worldwide had diabetes. ${ }^{2}$ A nationwide survey conducted by Yang et al in 2010 showed that the prevalence of diabetes and pre-diabetes among adults in China had reached 9.7\% and $15.5 \%$, respectively. ${ }^{3}$ As both pre-diabetes and type 2 diabetes (T2DM) have emerged as consequences of childhood obesity, ${ }^{4}$ the 
clustering of cardiovascular risk factors in this population $^{5}$ heightens concern that obese children and young adults are at risk for complications of diabetes, specifically cardiovascular disease (CVD). Thus, early identification of the population predisposed to developing diabetes is critically important if we are to target them for early intervention.

Screening for dysglycaemia (diabetes and pre-diabetes) has traditionally focused on OGTT to identify diabetes, impaired fasting glucose (IFG) and impaired glucose tolerance (IGT). However, due to time, expense and inconvenience, conducting an oral glucose tolerance test (OGTT) is often not feasible in patient care or population-based studies. ${ }^{6}$ Fasting blood glucose (FBG) has been used as an inexpensive alternative to the OGTT, but FBG is also associated with challenges, like the requirement for an 8-hour fast. In a study of diabetes screening practices among paediatric clinicians, a strong preference for non-fasting tests was evident. ${ }^{7}$

Haemoglobin A1c (HbA1c) has become increasingly popular for diabetes screening among primary care providers due to its many practical advantages including convenience of sampling, suitability as an index of chronic dysglycaemia, low intraindividual variability and propitious assay standardisation. ${ }^{8}$ In 2010, the American Diabetes Association (ADA) ${ }^{9}$ suggested that HbAlc values of $5.7 \%-6.4 \%$ established a diagnosis of pre-diabetes while a value of $\geq 6.5 \%$ defined diabetes. These recommendations are based on data in adults showing the relationship between HbAlc and the subsequent development of diabetic microvascular complications. However, it remains controversial what HbAlc level should be applied to the definition of pre-diabetes in children and adolescents, with at least three proposed thresholds: $6.0 \%,{ }^{10} 5.7 \%{ }^{9}$ and $5.5 \% .{ }^{11}$ Furthermore, it is unclear at what ages these HbA1c thresholds should be applied, due to the paucity of longitudinal data in children (and even young adults) which associate these cut points with adverse cardiometabolic outcomes. Until these longterm outcome data become available, pre-diabetes and diabetes can best be defined by their ability to identify pathophysiological abnormalities associated with hyperglycaemia such as decreased $\beta$-cell function and insulin sensitivity. ${ }^{12}$ Currently, studies in the Chinese paediatric population are lacking. Therefore, the aim of this study was to assess HbAlc as an instrument to establish the diagnosis of pre-diabetes and diabetes in a population of Chinese adolescents and young adults at increased risk of diabetes.

\section{MATERIALS AND METHODS \\ Subjects}

Subjects were recruited from the cohort of Beijing Children and Adolescents Metabolic Syndrome (BCAMS) study. The BCAMS is a longitudinal cohort study of cardiovascular risk factors since childhood. Details of the baseline study have been described previously. ${ }^{13}{ }^{14}$ Briefly, in 2004 a population-based survey was conducted in the Beijing area with a representative sample ( $\mathrm{n}=19593,50 \%$ boys) of schoolchildren (aged 6-18 years). Approximately 4500 subjects were identified as being at elevated risk for dysglycaemia at baseline due to the presence of one of the following risk factors: overweight defined by body mass index (BMI), total cholesterol $(\mathrm{TC}) \geq 5.2 \mathrm{mmol} / \mathrm{L}$, triglyceride $(\mathrm{TG}) \geq 1.7 \mathrm{mmol} / \mathrm{L}$ or FBG $\geq 5.6 \mathrm{mmol} / \mathrm{L}$ based on finger capillary blood tests. A follow-up study began in 2012 (8 years after baseline), with subjects recruited consecutively through various modalities (phone, text and email) for medical examination at Beijing Chaoyang Hospital. A total of 581 subjects who completed medical examination are included in this analysis. Those lost to follow-up were relatively younger and thinner at baseline than those who did follow-up; however, there were no significant difference in gender, pubertal status, blood pressure (BP), fasting TG, low-density lipoprotein cholesterol (LDL-C), high-density lipoprotein cholesterol (HDL-C) and FBG levels $(p>0.05)$. The BCAMS study was registered at www.clinicaltrials.gov (NCT03421444).

\section{Patient and public involvement}

No patients were involved in setting the research question or the outcome measures, nor were they involved in the design of this study. No patients were involved in the recruitment to and conduct of the study. There are no plans to disseminate the results of the research to study participants or the relevant patient community.

\section{Clinical measurements}

Height, weight, waist circumference (WC) and per cent body fat (FAT\%) were measured by trained field workers. Participants removed bulky clothing and shoes prior to measurements. Height was measured to the nearest $0.1 \mathrm{~cm}$ using a portable stadiometer. WC was measured midway between the lowest rib and the top of the iliac crest. Weight and FAT mass were measured to the nearest $0.1 \mathrm{~kg}$ using a Tanita Body Composition Analyzer (ModelTBF300A). Measurements of right arm systolic and diastolic BP (SBP and DBP) were performed 3 times $10 \mathrm{~min}$ apart and the mean values of the latter two measurements were recorded. BMI was calculated as weight divided by height squared.

\section{Laboratory measurements}

Venous blood samples were collected after an overnight ( $\geq 12$ hour) fast. An OGTT using $75 \mathrm{~g}$ glucose load was performed with plasma glucose levels in the fasting state (FBG), 0.5 hour and 2 hours (2hBG) measured using a hexokinase method. The concentrations of TG, TC and LDL-C were assayed using a standard enzymatic method. HDL-C was assessed with a phosphotungstic acid-Mg method. Serum C-reacting protein (CRP) was measured by immunoturbidimetric assay. Insulin concentrations were measured by monoclonal antibody-based sandwich enzyme-linked immunosorbent assays which 
was developed in the Key Laboratory of Endocrinology, Peking Union Medical College Hospital. The intraassay and interassay coefficients of variation for insulin were $<5.4 \%$ and $<9.0 \%$, respectively, with no cross-reactivity to proinsulin $(<0.05 \%)$. HbAlc was assayed using the TOSOH G7 automatic analysis system with high-pressure liquid chromatography. This assay is certified by the National Glycohemoglobin Standardization Program.

Insulin resistance was estimated by the following indices: (1) the homeostasis model assessment of insulin resistance (HOMA-IR) [(fasting insulin $\mathrm{mU} / \mathrm{L}) \times(\mathrm{FBG}$ $\mathrm{mmol} / \mathrm{L}) / 22.5]^{15}$; (2) Insulin Sensitive Index (Matsuda Index $), \quad[$ ISI $\quad$ (Matsuda $)=10000 / \sqrt{ }((\mathrm{FBG} \times$ fasting plasma insulin $) \times($ mean plasma glucose $\times$ mean plasma insulin)) $].{ }^{16}$ Pancreatic $\beta$-cell function was assessed by: (1) homeostasis model assessment of $\beta$-cell function (HOMA- $\beta$ ) $\left[(20 \times \text { fasting insulin) / (FPG-3.5) }]^{17}\right.$; (2) Insulinogenic Index (IGI= $\Delta$ insulin30/ $\Delta$ glucose 30$)$; (3) the ratio of the total area under the insulin curve to the total area under the glucose curve (total AUC insulin/glucose) and (4) the Oral Disposition Index $\left(\mathrm{DI}_{\mathrm{O}}=\mathrm{IGI} \times \mathrm{ISI}\right)$ which is the product of insulin sensitivity and insulin secretion. $^{18} 19$

\section{Definitions}

Dysglycaemia (IFG, IGT, pre-diabetes, diabetes) was defined according to current ADA guidelines. ${ }^{9}$ Metabolic syndrome (MS) was diagnosed according to 2009 Joint Task Force harmonisation criteria, with subjects exhibiting at least three of the following five components ${ }^{20}$ : (1) central obesity: WC $\geq 90$ th percentile for age and sex in $10-16$ years, or $\geq 90 \mathrm{~cm}$ for male and $\geq 80 \mathrm{~cm}$ for female; (2) IFG, IGT or diabetes; (3) BP: $\geq 130 / 85 \mathrm{~mm}$ $\mathrm{Hg}$; (4) HDL-C $<1.03 \mathrm{mmol} / \mathrm{L}$ in males, $<1.29 \mathrm{mmol} / \mathrm{L}$ in females and (5) TG $\geq 1.70 \mathrm{mmol} / \mathrm{L}$. According to Chinese age-specific and sex-specific BMI cut-offs, ${ }^{21}$ adolescents were classified as overweight if BMI was between the 85th and 95th percentile, and obese if BMI was above the 95th percentile. Subjects older than 18 years were classified overweight if $\mathrm{BMI} \geq 24 \mathrm{~kg} / \mathrm{m}^{2}$, or obese if $\mathrm{BMI} \geq 28 \mathrm{~kg} / \mathrm{m}^{2}$.

\section{Data analysis}

All statistical analyses were carried out using the Statistical Package for Social Sciences (SPSS V.19.0 for Windows). Continuous variables were tested for normality using a Kolmogorov-Smirnov test. Non-normal distribution values used in the analyses were log-transformed to improve normality. Results were expressed as mean $\pm \mathrm{SD}$. Group comparisons across three HbA1c categories were made with analysis of variance with Bonferroni post hoc comparison test. Agreement between HbAlc, fasting glucose category and OGTT 2-hour glucose was also assessed. K coefficients were reported. Receiver operating characteristic (ROC) curve analysis was performed for $\mathrm{HbAlc}$ and FBG to discriminate pre-diabetes from normal glucose tolerance (NGT) and T2DM, from NGT and IGT using a logistic procedure. Area under the curve (AUC) was considered as an effective measure of inherent validity of a diagnostic test. The mean values of variables were studied by analysis of variance. Multivariate logistic regression models were used to estimate ORs for IR, MS and its components. Level of significance was accepted as $\mathrm{p}<0.05$.

\section{RESULTS}

\section{Subjects characteristics}

The mean age of the entire population was $20.2 \pm 2.9$ years (female $46.8 \%$ ). The prevalence rates of obesity, high BP, dyslipidaemia and MS were $32.6 \%, 20.2 \%$, $29.5 \%$ and $14.5 \%$, respectively. Of 581 subjects, 18 refused to conduct 2-hour-OGTT. Using the HbA1c criteria recommended by ADA, the prevalence of T2DM was $1.5 \%(9 / 581)$ and pre-diabetes was $13.4 \%$ (78/581), whereas employing OGTT criteria yielded somewhat different prevalence rates (IFG 4.8\% (28/581), IGT $6.2 \%(35 / 563)$, IFG and/or IGT $10.1 \%(57 / 563)$ and T2DM $1.7 \%(10 / 581))$.

\section{Comparisons between $\mathrm{HbA1c}$ and fasting glucose}

The average HbA1c level was $5.4 \% \pm 0.6 \%$. HbAlc showed a strong positive correlation to FBG $(\mathrm{r}=0.734, \mathrm{p}<0.001)$, $2 \mathrm{hBG}(\mathrm{r}=0.694, \mathrm{p}<0.001)$, but a modest negative correlation with ISI $(\mathrm{r}=-0.177, \mathrm{p}<0.001)$, IGI $(\mathrm{r}=-0.258, \mathrm{p}<0.001)$ and $\mathrm{DI}_{\mathrm{O}} \quad(\mathrm{r}=-0.389, \mathrm{p}<0.001) \quad($ table 1$)$. There were also modest correlations between $\mathrm{HbAlc}$ and various cardiometabolic parameters, such as TG $(\mathrm{r}=0.159$, $\mathrm{p}<0.001)$, TC $(\mathrm{r}=0.157, \mathrm{p}<0.001)$, LDL-C $\quad(\mathrm{r}=0.176$, $\mathrm{p}<0.001)$, HDL-C $(\mathrm{r}=-0.103 \mathrm{p}<0.05)$, SBP $(\mathrm{r}=0.143$, $\mathrm{p}=0.001)$ and hsCRP $(\mathrm{r}=0.111, \mathrm{p}<0.05)$. FBG showed similar correlation to these cardiometabolic parameters, except for hsCRP ( $\mathrm{p}=0.125$ ).

The classification of subjects using $\mathrm{HbAlc}$ versus OGTT is shown in table 2. First, using OGTT data as a standard, the majority $(7 / 10,70.0 \%)$ of subjects with diabetes would have been diagnosed by $\mathrm{HbA1c} \geq 6.5 \%$. In contrast, only $25.7 \%(9 / 35)$ of subjects with IGT possessed elevated HbAlcs indicative of pre-diabetes, while the majority $(68.6 \%)$ had normal HbAlcs. Second, the majority $(87.6 \%)$ of the subjects with NGT would be identified with $\mathrm{HbAlc}<5.7 \%$, while $12.4 \%$ were classified with pre-diabetes or diabetes. On the other hand, of those considered to have diabetes by 2-hour OGTT (2hOGTT) criteria, 3 of $10(30.0 \%)$ were missed by HbA1c, while those identified as pre-diabetic on an OGTT (ie, IFG and/or IGT), 39 of 57 (68.4\%) were missed by HbA1c criteria.

However, of those diagnosed with diabetes using OGTT, only 4 of $10(40.0 \%)$ were identified by their FBG values, and among those with IGT, only 2 of 35 (5.7\%) were identified with IFG. So, using FPG criteria to identify dysglycaemia would miss the majority of IGT and T2DM and using HbAlc was only moderately better (as demonstrated by the low $\mathrm{k}$ coefficients between either FBG $(\mathrm{k}=0.16)$ or HbAlc $(\mathrm{k}=0.21)$ and OGTT. 


\begin{tabular}{|c|c|c|c|}
\hline & HbA1c & FBG & $2 \mathrm{hBG}$ \\
\hline HbA1c (\%) & 1 & $0.734^{\star \star}$ & $0.694^{\star \star}$ \\
\hline FBG (mmol/L) & $0.734^{\star \star}$ & 1 & $0.718^{\star *}$ \\
\hline $2 \mathrm{hBG}(\mathrm{mmol} / \mathrm{L})$ & $0.694^{\star \star}$ & $0.718^{\star \star}$ & 1 \\
\hline TG (mmol/L) & $0.159^{\star \star}$ & $0.182^{\star \star}$ & $0.196^{\star *}$ \\
\hline LDL-C (mmol/L) & $0.176^{\star *}$ & $0.108^{\star *}$ & $0.152^{\star *}$ \\
\hline TC (mmol/L) & $0.157^{\star \star}$ & 0.032 & 0.048 \\
\hline HDL-C (mmol/L) & $-0.103^{*}$ & $-0.095^{\star}$ & $-0.102^{*}$ \\
\hline SBP (mm Hg) & $0.143^{\star *}$ & $0.151^{\star \star}$ & $0.219^{\star \star}$ \\
\hline DBP (mm Hg) & $0.209^{\star \star}$ & $0.63^{\star \star}$ & $0.238^{\star *}$ \\
\hline MS score & $0.270^{\star *}$ & $0.215^{\star \star}$ & $0.326^{* *}$ \\
\hline Ln CRP (mg/L)† & $0.112^{*}$ & 0.069 & $0.126^{\star \star}$ \\
\hline Ln ISI† & $-0.177^{\star \star}$ & $-0.226^{\star \star}$ & $-0.304^{\star \star}$ \\
\hline Ln IGI† & $-0.258^{\star \star}$ & $-0.213^{\star \star}$ & $-0.282^{\star *}$ \\
\hline Ln DIo $†$ & $-0.389^{\star \star}$ & $-0.386^{\star *}$ & $-0.528^{\star *}$ \\
\hline Ln FINS (mU/L)† & $0.169^{\star \star}$ & $0.182^{\star \star}$ & $0.198^{\star \star}$ \\
\hline Ln 0.5hINS (mU/L)† & $-0.096^{\star}$ & $-0.121^{\star *}$ & -0.083 \\
\hline Ln 2hINS (mU/L)† & 0.038 & -0.025 & $0.357^{\star *}$ \\
\hline
\end{tabular}

MS score: numbers of MS components.

${ }^{*} \mathrm{P}<0.05$; ${ }^{* *} \mathrm{P}<0.01$.

†Log transformed before analysis.

0.5hINS, 0.5-hour insulin; 2hBG, 2-hour blood glucose; $2 \mathrm{hINS}$, 2-hour insulin; CRP, C-reacting protein; DBP, diastolic blood pressure; $\mathrm{DI}_{\mathrm{O}}$, Oral Disposition Index; FBG, fasting blood glucose; FINS, fasting insulin; HbA1c, haemoglobin A1c; HDL-C: highdensity lipoprotein cholesterol; IGI, Insulinogenic Index; ISI, Insulin Sensitivity Index; LDL-C: low-density lipoprotein cholesterol; Ln, Log transformed; MS, metabolic syndrome; SBP, systolic blood pressure; TC, total cholesterol; TG, triglycerides.

\section{ROC curve analysis}

Figure 1A,B represent the diagnostic accuracy of the HbAlc and FBG, for IGT and diabetes identified by OGTT, respectively. The AUC for HbAlc was 0.624
(95\% CI 0.582 to 0.664 ) and the AUC for FBG was 0.663 (0.576 to 0.749 ). The optimal HbA1c threshold for identifying IGT was $5.5 \%$, with a sensitivity of $42.9 \%$ and specificity of $78.6 \%$. To identify diabetes, the AUC for HbAlc was 0.970 (0.952 to 0.982), and for IFG the AUC was 0.789 (0.706 to 0.872 ). The optimal HbA1c threshold of $6.1 \%$, identified diabetes with $90.0 \%$ sensitivity and $98.7 \%$ specificity.

In light of the inconsistency between IFG and IGT when identifying dysglycaemia, we defined pre-diabetes as either IFG or IGT, and evaluated HbA1c test performance with ROC. As shown in figure $1 \mathrm{C}$ and table 3, the AUC of HbA1c for pre-diabetes was 0.680 (95\% CI 0.640 to 0.719 ), and the optimal threshold of HbAlc was still $5.5 \%$, with a sensitivity of $61.4 \%$ and specificity of $68.5 \%$. Moreover, as shown in figure 1 and table 3, compared with the ADA criteria for HbA1c (5.7\%), lowering the HbAlc threshold to $5.5 \%$ doubles the sensitivity of this test, while only moderately affecting the specificity.

\section{Comparisons of metabolic characteristics according to different HbA1c criteria}

To compare the segregation of metabolic characteristics among groups identified by our proposed $\mathrm{HbAlc}$ thresholds versus the ADA criteria, we stratified the population according to HbAlc categories (table 4). Age distribution, BMI, WC, DBP, FBG and 2hBG (all p<0.05) were all different among the three categories defined by either ADA or our proposed thresholds, while HDL-C was not. Not surprisingly, there were more subjects $(32.9 \%$ vs $13.4 \%$ ) classified as pre-diabetic based on HbA1c 5.5\%$6.1 \%$ than by ADA criteria of $5.7 \%-6.4 \%$. Similarly, a greater number of subjects $16(2.8 \%)$ vs $9(1.5 \%)$ would be considered to have T2DM by our criteria.

Table 4 compares the ability of these differing HbA1c strata to delineate levels of $\beta$-cell dysfunction and insulin resistance derived from FPG and OGTT measures. Subjects in the HbA1c $5.5 \%-6.1 \%$ vs $<5.5 \%$ categories

Table 2 The frequency of subjects with pre-diabetes and T2DM meeting the diagnostic criteria ( $\mathrm{HbA} 1 \mathrm{c}$, FBG and 2hBG after $75 \mathrm{~g}$-OGTT)

\begin{tabular}{|c|c|c|c|c|c|c|c|c|}
\hline \multirow[b]{2}{*}{ OGTT } & \multicolumn{3}{|l|}{ HbA1c* } & \multirow[b]{2}{*}{ Total } & \multicolumn{3}{|l|}{ FBG† } & \multirow[b]{2}{*}{ Total } \\
\hline & $\begin{array}{l}\text { NGT } \\
(<5.7 \%)\end{array}$ & $\begin{array}{l}\text { At risk for } \\
\text { diabetes } \\
(5.7 \%-6.4 \%)\end{array}$ & $\begin{array}{l}\text { T2DM } \\
(>6.4 \%)\end{array}$ & & $\begin{array}{l}\text { NGT } \\
\text { (<5.6 mmol/L) }\end{array}$ & $\begin{array}{l}\text { IFG } \\
(5.6-7.0 \mathrm{mmol} / \mathrm{L})\end{array}$ & $\begin{array}{l}\text { T2DM } \\
\text { (>7.0 } \mathrm{mmol} / \mathrm{L})\end{array}$ & \\
\hline NGT & 454 (87.6) & 64 (12.4) & 0 & 518 & 497 (95.9) & $21(4.1)$ & 0 & 518 \\
\hline IGT & 24 (68.6) & $9(25.7)$ & $2(5.7)$ & 35 & 33 (94.3) & $2(5.7)$ & 0 & 35 \\
\hline T2DM & $1(10.0)$ & $2(20.0)$ & $7(70.0)$ & 10 & $2(20.0)$ & $4(40.0)$ & $4(40.0)$ & 10 \\
\hline Total & 479 (85.1) & 75 (13.3) & $9(1.6)$ & $563 \ddagger$ & $532(94.5)$ & $27(4.8)$ & $5(0.7)$ & $563 \ddagger$ \\
\hline
\end{tabular}

Numbers in brackets are percentages of horizontal total.

${ }^{*}$ Kappa coefficient 0.21 .

†Kappa coefficient 0.16 .

$\ddagger 18$ of 581 subjects disagreed to undergo $2 \mathrm{hOGTT}$.

$75 \mathrm{~g}$-OGTT, $75 \mathrm{~g}$ oral glucose tolerance test; 2hBG, 2-hour blood glucose; 2h-OGTT, 2-hour oral glucose tolerance test; FBG, fasting blood glucose; HbA1c, haemoglobin A1c; IFG, impaired fasting glucose; IGT, impaired glucose tolerance; NGT, normal glucose tolerance; T2DM, type 2 diabetes. 

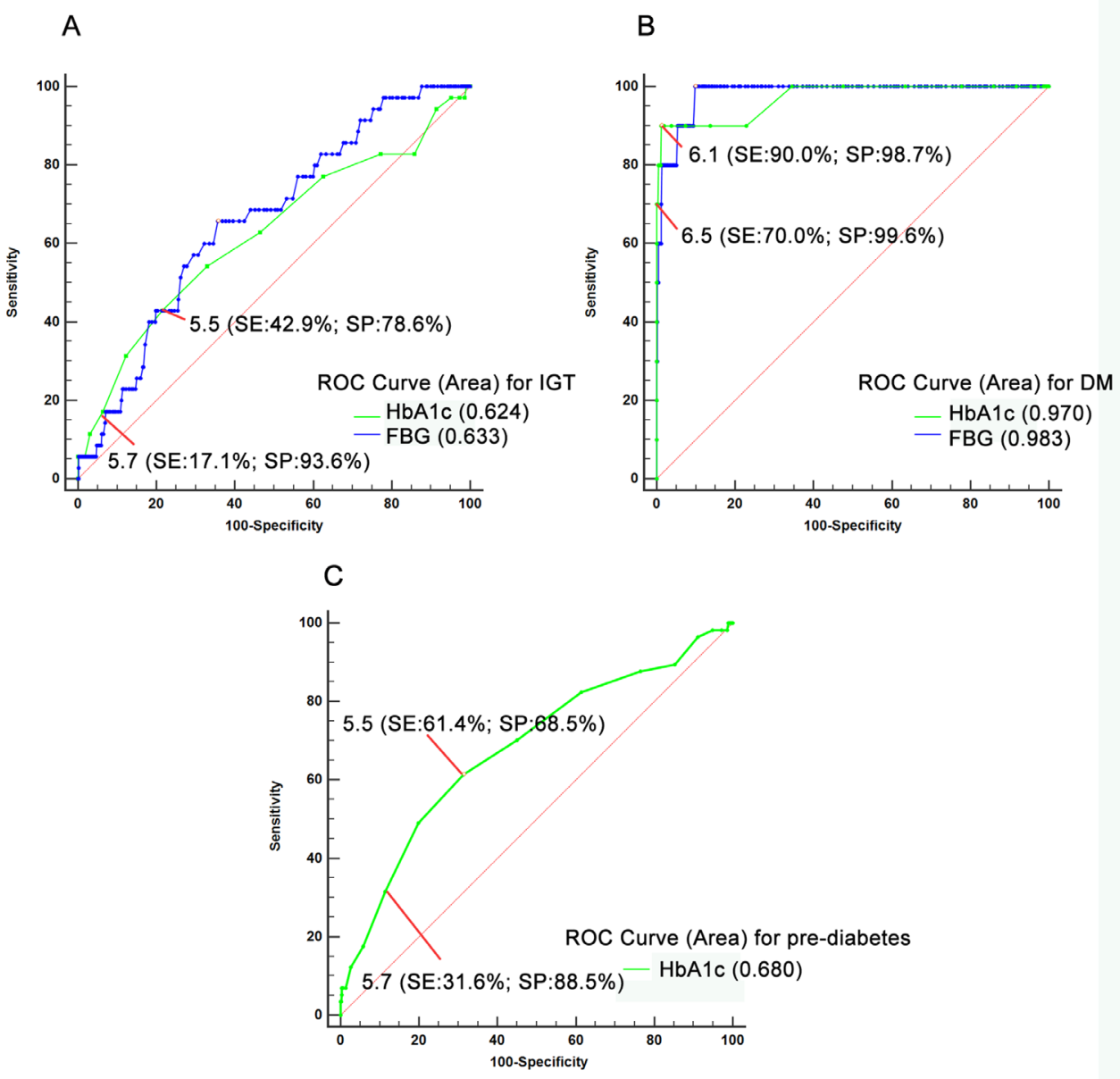

Figure 1 Comparison between the AUCs of the HbA1c and FBG for IGT (A) and T2DM (B) and the AUC of HbA1c for prediabetes $(\mathrm{C})$. The green discontinuous line indicates the curve defining the area for the HbA1c, and the blue continuous curve defines the area for FBG. Pre-diabetes was defined either by an FBG $\geq 5.6 \mathrm{mmol} / \mathrm{L}$ (IFG) or $2 \mathrm{hBG} \geq 7.8 \mathrm{mmol} / \mathrm{L}$ (IGT). The red arrows indicate the different thresholds (sensitivity, specificity) of HbA1c. 2hBG, 2-hour blood glucose; AUC, area under the curve; FBG, fasting blood glucose; HbA1c, haemoglobin A1c; IFG, impaired fasting glucose; IGT, impaired glucose tolerance; ROC, receiver operating characteristic curve; SE, sensitivity; SP, specificity; T2DM, type 2 diabetes.

demonstrated no difference with regard to insulin resistance indices (HOMA-IR and ISI), and neither did those with HbA1c 5.7\%-6.4\% comparing with those $<5.7 \%$. However, subjects with HbA1c $5.5 \%-6.1 \%$ showed a significantly lower IGI and $\mathrm{DI}_{\mathrm{O}}$ compared with those with HbAlc $<5.5 \%$. Notably, these differences were not as pronounced among groups classified using ADA criteria, especially with regard to IGI.

Regardless of the HbA1c thresholds employed, lipids measures (TC, TG and LDL-C) were significantly higher in diabetes than in normal or pre-diabetes categories, whereas hsCRP was highest in the pre-diabetic individuals $(\mathrm{p}<0.05)$. Moreover, high HbA1c was associated with a higher prevalence of obesity, hypertension, IR and dyslipidaemia. Of those with HbAlc 5.5\%-6.1\%, $18.8 \%$ had MS, compared with only $10.5 \%$ of those with HbA1c $<5.5 \%$. Applying our HbA1c thresholds (table 5), the ORs for dyslipidaemia, insulin resistance and MS in pre-diabetic versus non-diabetic individuals were $1.61,2.19$ and 2.09 , respectively, which is somewhat higher than if ADA criteria were employed.

\section{DISCUSSION}

This cross-sectional study demonstrates that the ADA's HbAlc thresholds of $5.7 \%$ and $6.5 \%$ had low sensitivity for classifying pre-diabetes $(31.6 \%)$ and diabetes $(63.6 \%)$ as defined by OGTT in a young Chinese population. Rather, we propose a threshold of $6.1 \%$ for identifying diabetes, with a sensitivity of $81.8 \%$ and specificity of $98.8 \%$, and $5.5 \%$ for detecting pre-diabetes, with a sensitivity of $68.5 \%$ and specificity of $61.4 \%$. We observed that the use of HbAlc thresholds of $5.5 \%$ and $6.1 \%$ would significantly improve the sensitivity of these measures without adversely affecting their specificities. Our data are in agreement with reports in children ${ }^{22-25}$ which have concluded that HbA1c thresholds of $5.7 \%-6.4 \%$ and $\geq 6.5 \%$ are insufficient to detect pre-diabetes and diabetes.

Several studies have assessed HbAlc cut points for predicting diabetes or pre-diabetes against the gold-standard OGTT and concluded that HbAlc is a poor predictor of pre-diabetes and T2DM in young people. Lee $e t a t^{23}$ examined the ability of various tests to diagnose pre-diabetes and diabetes in obese children; comparing HbAlc, 
Table 3 Test performance characteristics of specific $\mathrm{HbA1c}$ thresholds for detecting pre-diabetes and diabetes according to OGTT

\begin{tabular}{|c|c|c|c|c|c|c|}
\hline \multirow{2}{*}{$\begin{array}{l}\text { HbA1c } \\
\text { threshold }\end{array}$} & \multicolumn{3}{|c|}{ Pre-diabetes (IFG+IGT) } & \multicolumn{3}{|l|}{ T2DM } \\
\hline & Sensitivity & 1-specificity & Sensitivity+specificity & Sensitivity & 1-specificity & Sensitivity+specificity \\
\hline 2.7 & 1.000 & 1.000 & 1.000 & 1.000 & 1.000 & 1.000 \\
\hline 3.8 & 1.000 & 0.998 & 1.002 & 1.000 & 0.998 & 1.002 \\
\hline 4.0 & 1.000 & 0.996 & 1.004 & 1.000 & 0.996 & 1.004 \\
\hline 4.1 & 1.000 & 0.994 & 1.006 & 1.000 & 0.995 & 1.005 \\
\hline 4.3 & 1.000 & 0.992 & 1.008 & 1.000 & 0.993 & 1.007 \\
\hline 4.5 & 1.000 & 0.990 & 1.010 & 1.000 & 0.991 & 1.009 \\
\hline 4.7 & 0.982 & 0.986 & 0.997 & 1.000 & 0.986 & 1.014 \\
\hline 4.8 & 0.982 & 0.972 & 1.011 & 1.000 & 0.973 & 1.027 \\
\hline 4.9 & 0.982 & 0.950 & 1.033 & 1.000 & 0.953 & 1.047 \\
\hline 5.0 & 0.965 & 0.911 & 1.054 & 1.000 & 0.917 & 1.083 \\
\hline 5.1 & 0.895 & 0.853 & 1.042 & 1.000 & 0.857 & 1.143 \\
\hline 5.2 & 0.877 & 0.764 & 1.113 & 1.000 & 0.776 & 1.224 \\
\hline 5.3 & 0.825 & 0.615 & 1.210 & 1.000 & 0.635 & 1.365 \\
\hline 5.4 & 0.702 & 0.452 & 1.250 & 1.000 & 0.476 & 1.524 \\
\hline 5.5 & 0.614 & 0.315 & 1.300 & 1.000 & 0.344 & 1.656 \\
\hline 5.6 & 0.491 & 0.200 & 1.292 & 0.900 & 0.228 & 1.672 \\
\hline 5.7 & 0.316 & 0.115 & 1.201 & 0.900 & 0.136 & 1.764 \\
\hline 5.8 & 0.175 & 0.058 & 1.117 & 0.900 & 0.071 & 1.829 \\
\hline 5.9 & 0.123 & 0.026 & 1.097 & 0.900 & 0.036 & 1.864 \\
\hline 6.0 & 0.070 & 0.014 & 1.056 & 0.900 & 0.020 & 1.880 \\
\hline 6.1 & 0.070 & 0.006 & 1.064 & 0.900 & 0.013 & 1.887 \\
\hline 6.2 & 0.070 & 0.004 & 1.066 & 0.800 & 0.011 & 1.789 \\
\hline 6.3 & 0.053 & 0.004 & 1.049 & 0.800 & 0.009 & 1.791 \\
\hline 6.4 & 0.035 & 0.002 & 1.033 & 0.800 & 0.005 & 1.795 \\
\hline 6.5 & 0.035 & 0.000 & 1.035 & 0.700 & 0.004 & 1.696 \\
\hline 6.8 & & & & 0.700 & 0.000 & 1.700 \\
\hline 7.1 & & & & 0.600 & 0.000 & 1.600 \\
\hline 7.4 & & & & 0.500 & 0.000 & 1.500 \\
\hline 8.4 & & & & 0.400 & 0.000 & 1.400 \\
\hline 9.5 & & & & 0.300 & 0.000 & 1.300 \\
\hline 10.4 & & & & 0.200 & 0.000 & 1.200 \\
\hline 12.5 & & & & 0.100 & 0.000 & 1.100 \\
\hline 14.8 & & & & 0.000 & 0.000 & 1.000 \\
\hline
\end{tabular}

HbA1c, haemoglobin A1c; IFG, impaired fasting glucose; IGT, impaired glucose tolerance; OGTT, oral glucose tolerance test; T2DM, type 2 diabetes.

fructosamine and random glucose with OGTT. They found that all of these tests were poor discriminators, and led to missed cases of dysglycaemia in children. Similarly, Nowicka $e t a l^{44}$ suggested that an HbAlc of $5.8 \%$ for identifying T2DM in a multiethnic cohort of 1156 obese children and adolescents from the USA. Because of the lower prevalence of diabetes in paediatric as opposed to adult populations, the utility of A1c for detecting diabetes may be suspicious, and at the very least, thresholds require adjustment for the population under study. For this reason, Kester et $a l^{25}$ suggested caution when adopting $\mathrm{HbA1c}$ as a principal diagnostic method in children, and called for prospective studies of pre-diabetes and T2DM in obese paediatric populations to determine HbAlc cut-off points.

FBG has been used as an inexpensive alternative to OGTT, especially when screening for MS. In our study, we compared HbA1c with FBG to detect dysglycaemia. In the subjects categorised as pre-diabetic by OGTT, $31.6 \%$ showed laboratory evidence of being at risk for 
Table 4 Clinical features of the study population according to haemoglobin A1c categories

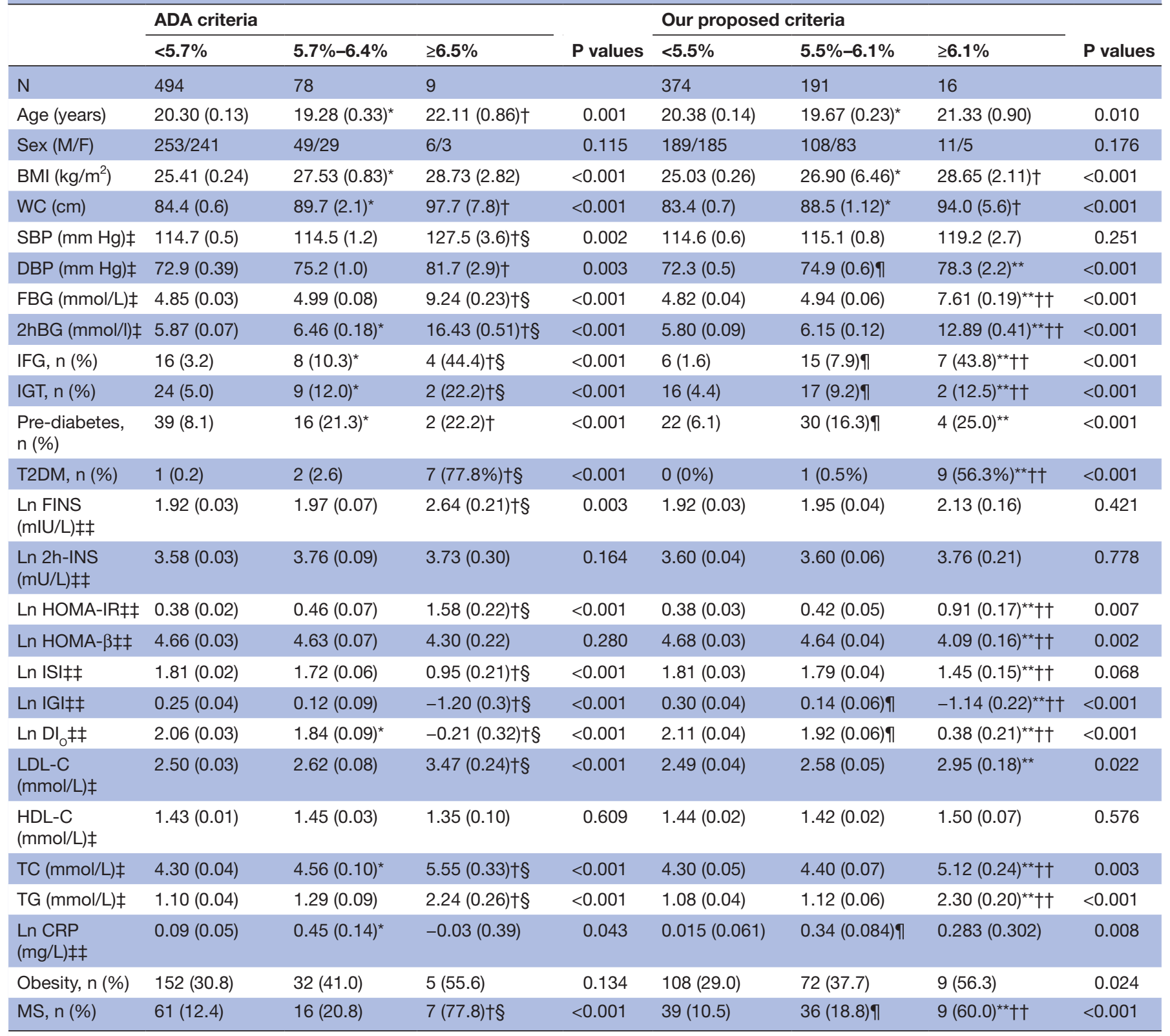

${ }^{*}<5.7 \%$ vs $5.7 \%-6.4 \%$.

$\dagger<5.7 \%$ vs $>6.4 \%$.

$\ddagger$ Adjusted for age, sex and BMI.

$\S 5.7 \%-6.4 \%$ vs $>6.4 \%$.

१ $<5.5 \%$ vs $5.5 \%-6.1 \%$.

** $<5.5 \%$ vs $>6.1 \%$

†† $5.5 \%-6.1 \%$ vs $>6.1 \%$.

¥¥Log transformed and adjust for age, sex and BMl; data were shown as mean (SE) or number (percentage).

2hBG, 2-hour blood glucose; 2hINS, 2-hour insulin; ADA, American Diabetes Association; BMI, body mass index; CRP, C-reacting protein; DBP, diastolic blood pressure; $\mathrm{DI}_{\mathrm{O}}$, Oral Disposition Index; FBG, fasting blood glucose; FINS, fasting insulin; HDL-C: high-density lipoprotein cholesterol; HOMA-IR, the index of homeostasis model assessment of insulin resistance; IFG, impaired fasting glucose; IGI, Insulinogenic Index; IGT, impaired glucose tolerance; ISI, Insulin Sensitivity Index; LDL-C: low-density lipoprotein cholesterol; MS, metabolic syndrome; SBP, systolic blood pressure; T2DM: type 2 diabetes; TC, total cholesterol; TG, triglycerides; WC, waist circumference.

$\mathrm{DM}$ on the basis of HbA1c, while only $5(6.2 \%)$ were identified with IFG. Of the 10 classified with DM by OGTT, seven subjects were detected with HbA1c, while only four would be identified with DM on the basis of FBG. These findings are consistent with Chan et al who showed that FPG performed poorly in obese adolescents and young adults aged 10-18years compared with HbAlc and OGTT when identifying dysglycaemia which was detected using a blinded continuous glucose monitoring device. ${ }^{26}$ Furthermore, HbA1c may present certain advantages over other tests. For example, the multiethnic healthy study cohort demonstrated greater 
Table 5 Age-adjusted and sex-adjusted ORs and 95\% Cls for IR, MS and its components according to haemoglobin A1c categories

\begin{tabular}{|c|c|c|c|c|c|c|c|c|}
\hline & \multicolumn{3}{|c|}{ ADA criteria } & \multirow{2}{*}{$\begin{array}{l}\text { P for } \\
\text { trend }\end{array}$} & \multicolumn{3}{|c|}{ Our proposed criteria } & \multirow{2}{*}{$\begin{array}{l}P \text { for } \\
\text { trend }\end{array}$} \\
\hline & $<5.7 \%$ & $5.7 \%-6.5 \%$ & $\geq 6.5 \%$ & & $<5.5 \%$ & $5.5 \%-6.1 \%$ & $\geq 6.1 \%$ & \\
\hline Elevated BP & 1 (ref) & $\begin{array}{l}1.55 \\
\text { (0.88 to } 2.74)\end{array}$ & $\begin{array}{l}17.75^{\star \star} \\
(3.26 \text { to } 96.76)\end{array}$ & 0.002 & 1 (ref) & $\begin{array}{l}1.37 \\
(0.89 \text { to } 2.15)\end{array}$ & $\begin{array}{l}5.63^{\star *} \\
(1.88 \text { to } 16.65)\end{array}$ & 0.006 \\
\hline Dyslipidaemia & 1 & $\begin{array}{l}1.53 \\
(0.91 \text { to } 2.57)\end{array}$ & $\begin{array}{l}5.02^{*} \\
(1.22 \text { to } 20.61)\end{array}$ & 0.026 & 1 & $\begin{array}{l}1.61^{*} \\
(1.10 \text { to } 2.37)\end{array}$ & $\begin{array}{l}3.34^{\star} \\
(1.17 \text { to } 9.54)\end{array}$ & 0.008 \\
\hline IR & 1 & $\begin{array}{l}2.10^{\star *} \\
(1.25 \text { to } 3.55 \square\end{array}$ & $\begin{array}{l}21.50^{\star *} \\
(2.56 \text { to } 180.56)\end{array}$ & $<0.001$ & 1 & $\begin{array}{l}2.19^{\star *} \\
(1.46 \text { to } 3.29)\end{array}$ & $\begin{array}{l}8.69^{*} \\
(2.54 \text { to } 29.70)\end{array}$ & $<0.001$ \\
\hline MS & 1 & $\begin{array}{l}1.95^{\star} \\
\text { (1.04 to } 3.64)\end{array}$ & $\begin{array}{l}20.80^{\star *} \\
(4.15 \text { to } 104.22)\end{array}$ & $<0.001$ & 1 & $\begin{array}{l}2.09^{\star \star} \\
(1.27 \text { to } 3.45)\end{array}$ & $\begin{array}{l}11.63^{\star \star} \\
(3.85 \text { to } 35.10)\end{array}$ & $<0.001$ \\
\hline
\end{tabular}

*Versus ref. $p<0.05 ;{ }^{* *}$ versus ref. $p<0.01$.

ADA, American Diabetes Association; BP, blood pressure; IR, insulin resistance, defined by the index of homeostasis model assessment of insulin resistance $>2.6$; MS, metabolic syndrome.

consistency of HbAlc versus FBG in a prospective trial in middle-school children. ${ }^{27}$

The debate over which test (HbA1c, FBG or 2hOGTT) is best to identify relevant glycaemic abnormalities in youth will ultimately require decades of prospective study to determine which test is most prognostic of cardiovascular and microvascular consequences. Until these longterm outcome studies become available, pre-diabetes and diabetes can be defined alternately by pathophysiological abnormalities associated with diabetes such as declining insulin sensitivity and $\beta$-cell function. Thus, the present study compares the ADA criteria with our proposed cut-off points to detect those alterations in insulin sensitivity and $\beta$-cell function based on OGTT. We demonstrate that an $\mathrm{HbAlc}$ in the range of $5.5 \%-6.1 \%$ is associated with reduced $\beta$-cell function (IGI, HOMA- $\beta$ ) as well as $\mathrm{DI}_{\mathrm{O}}$ which is an established metabolic predictor of progression to diabetes. ${ }^{28}$ We found progressively declining $\mathrm{DI}_{\mathrm{O}}$ across the continuum of HbA1c from $<5.5 \%$ to $5.5 \%-6.1 \%$ to $>$ $6.1 \%$. In contrast, the established ADA HbAlc criteria did not detect a difference in $\beta$-cell function (IGI) as HbAlc progressed from $<5.7 \%$ to $5.5 \%-6.4 \%$. This implies that our proposed thresholds are more rational for defining diabetes risk, at least in a young Chinese population.

Studies in adults and children have shown that pre-diabetes is associated with increased prevalence of cardiovascular risk factors. ${ }^{529}$ We also found a clustering of cardiovascular risk factors among subjects at risk for diabetes defined by our HbAlc thresholds. In the HbAlc $5.5 \%-6.1 \%$, compared with those $<5.5 \%$, elevated HbAlc was associated with known risk factors for CVD, including WC, DBP, TC, TG, LDL-C and hsCRP as well as a more than a twofold increased risk of having IR. Thus, our findings are consistent with an evolving consensus that HbA1c may identify a population with increased risk of microvascular and macrovascular complications.

CVD generally presents during adulthood, but the antecedents of this adult disease may be detectable in childhood. Elevated lipid and BP levels have been associated with an increased risk of CVD, and these risk factors track from childhood into adulthood. ${ }^{30}{ }^{31}$ Although this has not been definitively demonstrated in a prospective study of dysglycaemia which spans from childhood into adult life, it is reasonable to conclude that a similar persistence, if not a progression, of glycaemic abnormalities would be observed. Adolescents with pre-diabetes or T2DM face many years of hyperglycaemia and CVD, and thus, may have an increased life-time risk of developing complications. In fact, a large proportion of American adolescents have microalbuminuria and cardiovascular risk factors at diagnosis of T2DM. ${ }^{32}$

This study has several strengths and weaknesses. Considerable strength is drawn from the well-characterised cohort of adolescents and young adults at risk for diabetes. However, relatively few from this cohort had diabetes by ADA criteria. In addition, pubertal stage, which can influence insulin sensitivity and lipid levels, was not evaluated in this study. However, the small proportion of participants $(<12 \%)$ less than 16 years old suggests that the vast majority of participant were postpubertal, thus rendering this as only a minor concern. Furthermore, since puberty is associated with age, and we did include age as a covariate when comparing clinical features across HbAlc categories, there was some adjustment for the effect of pubertal stage. Finally, compared with our original population at baseline, the follow-up group is relatively small which may introduce the potential for bias. Nonetheless, there were no significant difference in gender, pubertal status or major cardiometabolic parameters at baseline between those who followed up versus those lost to follow-up.

In conclusion, the ADA's established HbAlc criteria for pre-diabetes and diabetes $(5.7 \%$ and $6.5 \%)$ may not be appropriately applied to adolescents and young adults, especially in the Chinese population. Our findings suggest that those with HbA1c of 5.5\%-6.1\% already exhibit impaired $\beta$-cell function and increased cardiometabolic risk which may warrant intervention. 
Author affiliations

'Department of Endocrinology, Key Laboratory of Endocrinology, National Health and Family Planning Commission, Peking Union Medical College Hospital, Chinese Academy of Medical Sciences and Peking Union Medical College (CAMS \& PUMC), Beijing, People's Republic of China

${ }^{2}$ Department of Endocrinology, Beijing Chaoyang Hospital, Capital Medical University, Beijing, People's Republic of China

${ }^{3}$ Epidemiology Department, Fielding School of Public Health, University of California Los Angeles, Los Angeles, California, USA

${ }^{4}$ Division of Endocrinology, The Children's Hospital of Philadelphia, Perelman School of Medicine, University of Pennsylvania, Philadelphia, Pennsylvania, USA

Acknowledgements We gratefully thank all participants of the BCAMS.

Contributors GL analysed data and drafted the manuscript; LH, YW, JF and YL contributed to data collection; $Y Z$ contributed to the data analysis and revised the manuscript; SMW was responsible for the concept and design of the study and contributed to the data interpretation and reviewed/edited the manuscript. ML contributed to the concept, design of the study, analysed the data and revised the manuscript. SG was responsible for the concept, design and data collection in the BCAMS follow-up study and contributed to acquisition and interpretation of the data, and revised the manuscript.

Funding This work was supported by Key Program of Beijing Municipal Science \&Technology Commission (D111100000611001, D111100000611002), National Key Research Program of China (2016YFC1304801), Beijing Natural Science Foundation (7172169), Beijing Science \& Technology Star Program (2004A027), Novo Nordisk Union Diabetes Research Talent Fund (2011A002) and National Key Program of Clinical Science (WBYZ2011-873)

Competing interests None declared.

Patient consent Obtained.

Ethics approval The study was approved by the Ethics Committee at the Beijing Chaoyang Hospital.

Provenance and peer review Not commissioned; externally peer reviewed.

Data sharing statement Additional details on data presented in the current study are available by emailing liming@pumch.cn or gaoshanmw@163.com.

Open access This is an open access article distributed in accordance with the Creative Commons Attribution Non Commercial (CC BY-NC 4.0) license, which permits others to distribute, remix, adapt, build upon this work non-commercially, and license their derivative works on different terms, provided the original work is properly cited, appropriate credit is given, any changes made indicated, and the use is non-commercial. See: http://creativecommons.org/licenses/by-nc/4.0/.

\section{REFERENCES}

1. Ng M, Fleming T, Robinson M, et al. Global, regional, and national prevalence of overweight and obesity in children and adults during 1980-2013: a systematic analysis for the Global Burden of Disease Study 2013. Lancet 2014;384:766-81.

2. Whiting DR, Guariguata L, Weil C, et al. IDF diabetes atlas: global estimates of the prevalence of diabetes for 2011 and 2030. Diabetes Res Clin Pract 2011;94:311-21.

3. Yang W, Lu J, Weng J, et al. Prevalence of diabetes among men and women in China. N Engl J Med 2010;362:1090-101.

4. Sinha R, Fisch G, Teague B, et al. Prevalence of impaired glucose tolerance among children and adolescents with marked obesity. $N$ Engl J Med 2002;346:802-10.

5. Di Pino A, Scicali R, Calanna S, et al. Cardiovascular risk profile in subjects with prediabetes and new-onset type 2 diabetes identified by $\mathrm{HbA}(1 \mathrm{c})$ according to American Diabetes Association criteria. Diabetes Care 2014;37:1447-53.

6. Sacks DB. A1C versus glucose testing: a comparison. Diabetes Care 2011;34:518-23.

7. Rhodes ET, Finkelstein JA, Marshall R, et al. Screening for type 2 diabetes mellitus in children and adolescents: attitudes, barriers, and practices among pediatric clinicians. Ambul Pediatr 2006;6:110-4.

8. Fajans SS, Herman WH, Oral EA. Insufficient sensitivity of hemoglobin $\mathrm{A}(\square \mathrm{C})$ determination in diagnosis or screening of early diabetic states. Metabolism 2011;60:86-91.
9. American Diabetes Association. Diagnosis and classification of diabetes mellitus. Diabetes Care 2010;33 Suppl 1(Suppl 1):S62-9.

10. Gillett MJ. International Expert Committee. International Expert Committee report on the role of the $\mathrm{A} 1 \mathrm{C}$ assay in the diagnosis of diabetes. Diabetes Care 2009;32:1327-34.

11. Expert Committee on the Diagnosis and Classification of Diabetes Mellitus. Report of the expert committee on the diagnosis and classification of diabetes mellitus. Diabetes Care 2003;26(Suppl 1):S5-20.

12. Sjaarda LA, Michaliszyn SF, Lee $\mathrm{S}$, et al. $\mathrm{HbA}(1 \mathrm{c})$ diagnostic categories and $\beta$-cell function relative to insulin sensitivity in overweight/obese adolescents. Diabetes Care 2012;35:2559-63.

13. Li M, Fisette $A$, Zhao $X Y$, et al. Serum resistin correlates with central obesity but weakly with insulin resistance in Chinese children and adolescents. Int J Obes 2009;33:424-39.

14. Li L, Yin J, Cheng H, et al. Identification of Genetic and Environmental Factors Predicting Metabolically Healthy Obesity in Children: Data From the BCAMS Study. J Clin Endocrinol Metab 2016;101:1816-25

15. Yin J, Li M, Xu L, et al. Insulin resistance determined by Homeostasis Model Assessment (HOMA) and associations with metabolic syndrome among Chinese children and teenagers. Diabetol Metab Syndr 2013;5:71.

16. Monzillo LU, Hamdy O. Evaluation of insulin sensitivity in clinical practice and in research settings. Nutr Rev 2003;61:397-412.

17. Matthews DR, Hosker JP, Rudenski AS, et al. Homeostasis mode assessment: insulin resistance and beta-cell function from fasting plasma glucose and insulin concentrations in man. Diabetologia 1985;28:412-9.

18. Kahn SE, Prigeon RL, McCulloch DK, et al. Quantification of the relationship between insulin sensitivity and beta-cell function in human subjects. Evidence for a hyperbolic function. Diabetes 1993;42:1663-72.

19. Ram J, Snehalatha C, Selvam S, et al. The oral disposition index is a strong predictor of incident diabetes in Asian Indian prediabetic men. Acta Diabetol 2015;52:733-41.

20. Alberti KG, Eckel RH, Grundy SM, et al. Harmonizing the metabolic syndrome: a joint interim statement of the International Diabetes Federation Task Force on Epidemiology and Prevention; National Heart, Lung, and Blood Institute; American Heart Association; World Heart Federation; International Atherosclerosis Society; and International Association for the Study of Obesity. Circulation 2009;120:1640-5.

21. Group of China Obesity Task Force. [Body mass index reference norm for screening overweight and obesity in Chinese children and adolescents]. Zhonghua Liu Xing Bing Xue Za Zhi 2004;25:97-102.

22. Lee JM, Gebremariam A, Wu EL, et al. Evaluation of nonfasting tests to screen for childhood and adolescent dysglycemia. Diabetes Care 2011;34:2597-602.

23. Lee JM, Wu EL, Tarini B, et al. Diagnosis of diabetes using hemoglobin A1c: should recommendations in adults be extrapolated to adolescents? J Pediatr 2011;158:947-52.

24. Nowicka $\mathrm{P}$, Santoro $\mathrm{N}$, Liu $\mathrm{H}$, et al. Utility of hemoglobin $\mathrm{A}(1 \mathrm{c})$ for diagnosing prediabetes and diabetes in obese children and adolescents. Diabetes Care 2011;34:1306-11.

25. Kester LM, Hey H, Hannon TS. Using hemoglobin A1c for prediabetes and diabetes diagnosis in adolescents: can adult recommendations be upheld for pediatric use? J Adolesc Health 2012;50:321-3.

26. Chan CL, Pyle L, Newnes L, et al. Continuous glucose monitoring and its relationship to hemoglobin A1c and oral glucose tolerance testing in obese and prediabetic youth. J Clin Endocrinol Metab 2015;100:902-10.

27. Buse JB, Kaufman FR, Linder B, et al. Diabetes screening with hemoglobin $A(1 c)$ versus fasting plasma glucose in a multiethnic middle-school cohort. Diabetes Care 2013;36:429-35.

28. D'Adamo E, Caprio S. Type 2 diabetes in youth: epidemiology and pathophysiology. Diabetes Care 2011;34(Suppl 2):S161-5

29. Shah AS, Gao Z, Urbina EM, et al. Prediabetes: the effects on arterial thickness and stiffness in obese youth. J Clin Endocrinol Metab 2014;99:1037-43.

30. Webber LS, Srinivasan SR, Wattigney WA, et al. Tracking of serum lipids and lipoproteins from childhood to adulthood. The Bogalusa Heart Study. Am J Epidemiol 1991;133:884-99.

31. Fuentes RM, Notkola IL, Shemeikka S, et al. Tracking of systolic blood pressure during childhood: a 15-year follow-up populationbased family study in eastern Finland. J Hypertens 2002;20:195-202.

32. Copeland KC, Zeitler P, Geffner M, et al. Characteristics of adolescents and youth with recent-onset type 2 diabetes: the TODAY cohort at baseline. J Clin Endocrinol Metab 2011;96:159-67. 